\title{
The long-term concerns post cochlear implantation as experienced by parents/caregivers of prelingually deaf children between the ages of 3 and 5 years in Gauteng Province, South Africa
}

\author{
N Moroe, MA (Audiology); N Kathrada, (BA) Speech and Hearing Therapy \\ Speech Therapy and Audiology Department, School of Human and Community Development, Humanities Faculty, University of the \\ Witwatersrand, Johannesburg, South Africa
}

Corresponding author: N Moroe (nomfundo.moroe@wits.ac.za)

\begin{abstract}
Background. Cochlear implantation aims to provide an effective means of spoken communication for prelingually deaf children. However, studies in this field are mostly clinically orientated, with little focus on the experiences and long-term concerns of families post cochlear implantation (CI).

Objective. To describe the long-term concerns post $\mathrm{CI}$ as experienced by parents/caregivers of prelingually deaf children between the ages of 3 and 5 years, and to determine the role of support groups with regard to effective intervention and coping post CI.

Methods. A phenomenological, non-experimental research design was conducted through semi-structured, indepth, one-on-one interviews with five parents/caregivers.

Results. Caregivers reported concerns with changes in family support, financial difficulties, poor communication, and schooling and vocational prospects for their children.

Conclusion. The findings of this study highlight a need for continued support for parents and families with children who have been fitted with cochlear implants. This should be through the use of a family systems perspective model that takes into account the impact on the quality of life of families with children who have a hearing loss or who are fitted with cochlear implants. There is a need for a contextualised longitudinal study where, based on previous observations and experiences, parents/caregivers are reminded about the continual long-term expenses associated with CI. This includes maintenance costs, hospital visits and school placement when the children reach school-going age.
\end{abstract}

S Afr J Child Health 2016;10(2):126-129. DOI:10.7196/SAJCH.2016v106i7.1049

Studies on cochlear implantation (CI) have mostly highlighted the rollercoaster emotions experienced by families when a diagnosis of a hearing impairment is made. ${ }^{[1]}$ The experiences of caregivers post CI have largely been neglected. For this article, the term caregiver refers to the natural parents or any family members who may assume the role of caring for a child who may not be their biological child. ${ }^{[2]}$ In South Africa (SA) many children are being raised by grandparents mostly due to divorce, substance abuse, child abuse/neglect, HIV/AIDS, unemployment or death. ${ }^{[3,4]}$ Furthermore, SA is a developing country characterised by high rates of poverty, unemployment, poor access to healthcare facilities and a high burden of disease. ${ }^{[5]}$ There are also discrepancies in gender equality where women, often grandparents, are the ones who assume the caregiver role ${ }^{[6]}$ and in most cases, these grandparents are above the age of employment, thereby relying on government's pension grant for income ${ }^{[3]}$ Consequently, caregivers inherit the 'caregiver burden, ${ }^{\text {' }}{ }^{[7]}$ described as the 'consequences of the activities involved in providing necessary direct care to a relative or friend that result in observable and perceived costs to the caregiver. ${ }^{[7]}$ In this case the caregiver burden is associated with raising a child with cochlear implants.

\section{Background}

Globally, 6 in 1000 babies are born with a hearing impairment. ${ }^{[8]}$ This affects language acquisition, since language development relies heavily on hearing. ${ }^{[9]}$ Unless hearing impairment is identified and managed early, a child with a hearing impairment may miss an opportunity to develop spoken language. ${ }^{[9]}$ Although SA is a developing country with access to developed world technology, ${ }^{[5]}$ the public healthcare sector is marred by poor service delivery, and this affects the early detection and management of hearing impairments. ${ }^{[5]}$ While rehabilitative services are essential, they are not a high priority when compared with life-threatening diseases such as tuberculosis (TB) or HIV/AIDS. ${ }^{[10]}$ Cochlear implants are highly recommended in children with severe-to-profound sensorineural loss, ${ }^{[11]}$ and who meet the candidacy criteria as discussed by Gray et al. ${ }^{[12]}$ However, cochlear implants are costly. Kerr et al ${ }^{[10]}$ found that in the first 10 years of implantation, the average estimated cost for a paediatric CI was ZAR455 225. This figure may have since increased, affecting families with children who are fitted with cochlear implants. The CI process is also lengthy and emotionally draining. According to Luterman, ${ }^{[13]}$ having a child with a hearing impairment creates stress within the family owing to continuous decisions such as suitable schools and the mode of communication for the family. There are $\sim 10$ schools in Gauteng Province, SA, that cater for children with hearing impairments (Peel EL. Inclusive Practice in South Africa. A Deaf Education Perspective. 2004: unpublished). The mode of communication offered and the proximity of the school may cause a concern for parents. Parents may enrol children at boarding schools or alternatively choose a local mainstream school or a school for learners with special educational needs. These often fail to cater for a child with cochlear implants.

\section{Methods}

A purposive sampling strategy was used to recruit three biological mothers and two caregivers. Parents/caregivers of children who were prelingually deafened between the ages of 3 and 5 years, fitted with cochlear implants and currently attending paediatric aural rehabilitation (AR), were recruited (Table 1). 
Table 1. Participants' profiles

\begin{tabular}{|c|c|c|c|c|c|}
\hline Participants & 1 & 2 & 3 & 4 & 5 \\
\hline Relationship to child & Mother & Mother & Foster parent & Mother & Grandmother \\
\hline Race & Black & Black & Black & Indian & Black \\
\hline $\begin{array}{l}\text { Gender of parent / } \\
\text { caregiver }\end{array}$ & Female & Female & Female & Female & Female \\
\hline Home language & Sesotho & Tshivenda & isiZulu & English & isiZulu \\
\hline $\begin{array}{l}\text { Language interview } \\
\text { was conducted in }\end{array}$ & Sesotho & English & English & English & English \\
\hline Residence & Johannesburg south & Johannesburg south & Johannesburg south & Johannesburg south & Johannesburg south \\
\hline Employment & Unemployed & Employed & Employed & Employed & Unemployed \\
\hline Education & - & Tertiary & Grade 11 & Tertiary & Diploma \\
\hline Children with CI, $n$ & 1 & 2 & 1 & 1 & 1 \\
\hline $\begin{array}{l}\text { Aetiology of hearing } \\
\text { impairment }\end{array}$ & Unknown & Waardenburg syndrome & Meningitis & Ototoxicity & Unknown \\
\hline Age of child (years) & 4 & 8 (child 1$), 2.5$ (child 2$)$ & 5 & 5 & 3 \\
\hline Gender of child & Female & Female (child 1), male (child 2) & Male & Male & Male \\
\hline AR & Yes & Yes & Yes & Yes & Yes \\
\hline CI support group & No & Yes & Yes & No & Yes \\
\hline
\end{tabular}

The study received ethical clearance from the University of the Witwatersrand Human Research Ethics Committee (non-medical) (clearance certificate number: H14/03/16). Once clearance had been attained, a Speech Therapy and Audiology Department of a local hospital in Johannesburg, which offers paediatric AR, was approached for possible participants. A proposal of the study was submitted to the hospital's ethics committee for approval and permission to conduct the study was granted. The participants were approached through the audiologist responsible for the paediatric AR clinic. The audiologist identified possible participants, informed them about the study and requested their permission to forward their contact details to the researcher. The participants were contacted telephonically to request their permission to participate in the study. Initially, eight participants were contacted; two participants declined and another participant did not meet the inclusion criteria. The remaining five participants were invited to participate in an interview.

One-on-one, open-ended interviews were conducted to collect data. The participants were interviewed at the hospital when they were attending their paediatric AR appointment. On the day of the interview, participants were given the information letter and a consent letter to sign. Permission to use a tape-recorder was also requested. The interviews were approximately 60 minutes long and were conducted in English for four participants and SeSotho for the fifth. The interview conducted in SeSotho was translated into English for transcription.

Informed consent, autonomy and confidentiality were discussed with the participants. Anonymity was maintained by assigning a code system instead of participant names. Participants were made aware that they were free to choose not to participate or withdraw from the study at any time without any negative consequences. There were no identified or assumed risks associated with this study nor were there any immediate benefits related.

Thematic analysis as outlined by Altschuld and White ${ }^{[14]}$ was used to analyse the themes that emerged, which were: unrealistic expectations, family support, education, communication, hidden financial costs and vocational prospects.

\section{Results}

All the participants expressed that they were informed that AR is a long process. Despite this, they still had unrealistic expectation with regards to cochlear implants.

'I thought in 6 months or something everything will be fine and she will speak, but they (the therapists) explained to me again.' - P1

'I thought after few days he would say I'm hungry or I love you but I understand it's a process now. - P3

However, with support and debriefing with the audiologists, the process was explained again and the participants were able to reconstruct realistic expectations.

'Now I feel like I am able to deal and accept the challenges, it is just one obstacle that I can overcome.' - P4

All participants agreed that family support was even more crucial post CI, but there were mixed feelings from their families. Two participants stated that initially they sought advice from the elders in the family. However, when they did not receive support, they made their own decisions.

'They didn't want to accept the hearing loss or the cochlear implants. They gave support after a long long time. We first went to traditional methods to ease the family but the father and I chose cochlears. For my son it was easier because I knew what to do.' - P2

One participant stated that cultural differences affected decision making.

'They are very Zulu. Very cultural and they were in denial. They said that there is no one in their family who is disabled. And so they blamed my daughter. But we had hope and we could see changes after cochlears. So their denial was their own.' - P5

Another participant revealed that her disagreements with her partner delayed intervention and eventually this led to a permanent separation.

'We are not together anymore. It was too much fighting and no support.' - P2 
All participants expressed difficulty in finding a crèche or schools as many schools rejected their children due to the school's lack of understanding of cochlear implants.

'No crèche wanted to take him. They just hear 'hearing loss' and they say no.' - P2

Three participants visited schools for learners with special educational needs were unhappy with the quality of education and the availability of resources.

'Remedial schools close to our home seemed to cater for many children with disabilities but nothing seemed to be done to actually assist their needs. Also, I didn't think putting him in a special needs school was best. He is okay academically, he just has a language delay'. - P4

Participant 3 revealed that despite the school claiming to accept her child, she noticed various inconsistencies in the school.

'I don't know if what they say is what they do. Last time I forgot to pack his battery, the cochlear battery for two or three days because I was working overtime and came home very late. My sister used to pack my son's lunch and all that. But anyways I realised there was no questions about the battery from the school and that simply means they are not putting his cochlears on or checking.' - P3

All the participants also expressed private schools were well equipped to cater for their children; however, they were unaffordable.

'There are schools in the North, remedial schools. But economically there is no way a normal man can afford those schools. It was R5 000 a month when we asked, that does not include spending or travelling costs.' - P1

Participants felt the government should play a role in ensuring fair and equal education for children with cochlear implants.

'The government should include courses, not special ones, but for all teachers so that they know how to help children with disability or needs.' - P5

All participants highlighted that they are concerned with the mode of communication used at home.

'Language is a major problem. His vocabulary is extremely limited. We always try to correct his speech and language. Up to now he always forgets to say the last part of a word.' - P2

'People don't know about sign language and everything. There are no opportunities for us. Even I can see that I am failing my son. There is no communication for him until the next morning at school. I really am failing my son.' - P3

Participants indicated that owing to poor communication at home, some children present with challenging behaviours.

'I am worried about his behaviour. He gets angry quickly because we don't understand him most of the time. The message isn't put across.' - P4

'He throws a lot of tantrums, he is always frustrated with the long journey, the communication and also with learning in therapy.' - P2

All participants commented that poor communication has negatively affected their children's social wellbeing.

'He doesn't have a friend, especially one who is deaf or uses Sign Language. He only communicates at school.' - P1

Participants with other hearing children also stated that one of the challenges they face is ensuring equality in the family. It is easy for their implanted child to be left out of conversations and have limited participation in the family. All the participants agreed that they were informed of the ongoing therapy sessions and device maintenance post CI; however, four participants stated that their financial circumstances have since changed.

'I couldn't work because no one would give me off every week to come for therapy. But now I am going to try and look for a job. I've been out of work because we have to come to therapy but my husband can't work alone now. We don't have a grant.' - P1

'The therapists really like me to come every week but I don't have means. I don't have financial ...uh... to come every week cause if I come here I have to be out of work.' - P3

All participants also commented on the maintenance of the cochlear implant device.

'It is not a cheap thing to maintain, but then we obviously went into it knowing this. But you don't realise it until you are placed into the situation and then you're like woah this is an expensive device.' - P4

'And life is very expensive. Transport is like R700 a month. He have to eat. He have to be warm. He have to have medication for flu whatever, you see. And school is R7 500. We do save and tell him to be careful, but he is a child, a boy, he has to play.' - P3

Two participants are currently receiving the state's social grant, while two participants are not eligible for a grant and one has not applied for a grant. The participants who receive the social grant indicated that even though the grant helps, it is not enough as life is very expensive.

Participants expressed concerns regarding vocational prospects and the future for their children.

'I have many concerns for the future. It worries me every day. My most important concern is his education. I think I may have to take him out of school at the first point of exit which is grade nine. Don't you think he'll need to go into a career in which he has to do something hands on instead of education?' - P1

'I want him to grow up and do things for himself. I don't want to close the doors for him.' - P3

'Right now we are there to assist him with finances, in the long term going into the work force with a severe disability will he be able to maintain those cochlears? The parts are ridiculously priced. It's thousands for repairs uh... parts and batteries and a hearing disability means a lower income.' - P4

\section{Discussion}

The findings suggest that parents/caregivers had unrealistic expectations regarding $\mathrm{CI}$, as some parents expected their children to talk instantly post CI. When these expectations were not met, the parents/caregivers experienced disappointment. Parents are active participants in the CI process, but because the process is lengthy, they may start having unrealistic expectations and may forget the discussions they had with the audiologists regarding realistic expectations. Therefore parents and audiologists need to remind each other about the benefits, limitations and the process of CI. Cohen ${ }^{[15]}$ asserts that constant provision of information at every stage allows parents to view the ongoing CI process in a rational and reasonable manner. Furthermore, these findings highlight the importance of constant feedback to prevent the unresolved feelings from resurfacing. Tye-Murray ${ }^{[16]}$ cautions that grief is not experienced in a sequential manner and parents may not necessarily resolve all negative emotions and experiences. As the child grows and interacts in different social contexts, it may trigger unresolved feelings for the parents. It is therefore necessary for parents to have information and a support base from professionals and family members as they go through the CI process.

Participants had mixed feelings regarding the availability of support from family members and audiologists. Throughout this journey, it is crucial for parents/caregivers to have a support base as they are faced with making informed choices regarding intervention, mode of communication and educational choices for the child. ${ }^{[17]}$ 
Most et al. ${ }^{[17]}$ states that each family is different, and may implement different coping strategies; however, the presence of a hearing impairment may cause instability in the family. There is a need for continued assessment to establish the support and emotional status of the family. Jackson and Turnbull ${ }^{[18]}$ assert that the presence of a hearing impairment in any family can negatively affect the wellbeing of the family. Therefore, CI team members, through the family systems perspective model, need to adopt a holistic view of the family, so as to take into account the family interactions, resources, parenting and support for a child with a hearing impairment. ${ }^{[18]}$

From the outset, despite the fact that participants were aware of the financial burden associated with cochlear implants, they were not fully prepared to meet the ongoing financial demands post CI. Kerr et al. ${ }^{[10]}$ advise that cochlear implants candidates should be informed about the ongoing costs and the likely time periods post CI as this knowledge will assist in assessing the affordability of intervention and planning for the long-term successful use of implants. Audiologists should realistically consider the candidate's ability to afford cochlear implants so that candidates are not unintentionally burdened beyond their affordability to achieve good results. Adopting the family system perspective model may assist in identifying the financial burdens the parents may be facing and how best the family can be assisted. ${ }^{[18]}$ It is worth noting that audiologists may not be in a position to financially assist the family with ongoing costs of cochlear implants. They can, however, assist through referrals to other services such as counselling or social services for social grants (if they haven't been referred). Furthermore, audiologists can implement a contextualised longitudinal study where experiences of other parents/caregivers who have engaged in the CI process can be shared with the parents enrolling in this programme. These experiences may be shared to provide insight into the continual long-term expenses and challenges associated with CI.

Participants felt that the schools were either not well equipped or were inconsistent around how they provided access to children with cochlear implants. Furthermore, schools that adequately cater for children with cochlear implants were expensive. De Villiers ${ }^{[19]}$ said that the limited number of schools for children with hearing impairment is largely due to the government's failure in the education and training of people with this affliction. This failure has left children with hearing impairment with vocational education as one of the available limited options. The South African education system and school programmes do not seem to promote or empower children with hearing impairment to be productive and working citizens of SA. ${ }^{[20]}$ Ultimately, it is the child with a hearing loss who suffers as they are unable to access good schools due to their high cost. This calls for policies regarding the quality of education for children with hearing impairment as poor quality education has lifetime effects on the vocational prospects for the hearing-impaired children and their parents.

The findings of this study also highlighted the parents/caregivers' plight with regard to communication at home. This is largely due to the different modes of communication used in the home. The parents/caregivers were more concerned that their children developed behavioural problems and were socially isolated by their peers due to restricted communication between them and their peers. This difficulty in communication may be expected as an ineffective mode of communication between caregiver and child can lead to parental stress, which consequently may lead to negative developmental outcomes as well as behavioural problems in the child with hearing impairments. ${ }^{[13]}$ These challenges can also be addressed through the family systems perspective models, where support for a child with a hearing impairment is taken into consideration. In this case, support may speak to access to services such as AR, which may help with communication strategies for the family, support for the child to make friends and inclusion of the child in the community. ${ }^{[18]}$ Since this model takes into account the wellbeing of the whole family, it will go a long way in ensuring that the needs of a family as a whole are addressed.

\section{Conclusion}

There is a need to adopt a holistic model that takes into account the wellbeing of family with a child fitted with cochlear implants. Jackson and Turnbull ${ }^{[18]}$ created a family systems perspective model which addressed the impact of deafness and the quality of life in the family. This model highlights the importance of family interactions, family resources, parenting and support for the child. Such a model will assist in evaluating the financial affordability of the parents/caregivers as they continue with the CI process. Furthermore, audiologists working or interacting with parents/caregivers of children with cochlear implants need to embrace the family systems perspective model to ensure that families have access to services that they may require as a family. These services may include referrals to other professionals such as counsellors to address issues expressed by parents/caregivers with regards to the lack of support from their families and finding suitable schools. Lastly, multidisciplinary professionals should provide continual screening and support throughout the intervention, as familyies financial and support needs may change or progress over time. This may be achieved through conducting a contextualised longitudinal study where the experiences of other parents/caregivers who have engaged in the process may be shared with the parents/ caregivers who are enrolled in the programme.

\section{References}

1. Anagnostou F, Graham J, Crocker S. A preliminary study looking at parental emotions following cochlear implantation. Cochlear Implants Int 2007;8(2):6886. DOI:10.1002/cii.331

2. Bouvard M. Mothers in All but Name: Grandmothers, Aunts, Sisters, Friends, Strangers, Nannies. Nicholasville, Kentucky: Wind Publications, 2009.

3. Park H. Grandmothers raising grandchildren: Family well-being and economic assistance. Focus 2005;24(1):19-27.

4. Turner J. Grandparents Raising Grandchildren. Washington DC: American Association of Retired Persons, 2005

5. Störbeck C, Moodley, S Cochlear Implantation in an Early Intervention Programme in South Africa. Conference Proceedings: 10th European Symposium on Paediatric Cochlear Implantation, 12-14 May 2001, Athens.

6. Barrow M. Aging, the Individual and Society. 6th ed. London: West Publishing Company, 1999.

7. Hunt CK. Concepts in caregiver research. J Nurs Sch 2003;35(1):27-32. DOI:10.1111/j.1547-5069.2003.00027.x

8. Swanepoel D, Störbeck C, Friedland P. Early hearing detection and intervention in South Africa. Int J Pediatr Otorhinolaryngol 2009;73(6):783-786. DOI:10.1016/j. ijporl.2009.01.007

9. Clark EV. First Language Acquisition. 2nd ed. Cambridge: Cambridge University Press, 2009.

10. Kerr G, Tuomi S, Müller A. Costs involved in using a cochlear implant in South Africa. S Afr J Commun Disord 2012;59(1):16-26. DOI:10.7196/SAJCD.117

11. Le Roux T, Laurent C. Open access guide to audiology and hearing aids for otolaryngologists. Cape Town: University of Cape Town, 2013.

12. Gray RF, Jones F, Shipgood L, Court I. Paediatric cochlear implantation: The balance between professional caution and urgency of treatment. Cochlear Implants Int 2003;4(1):45-51. DOI:10.1179/cim.2003.4.1.45

13. Luterman D. Counselling parents about cochlear implants. The American Speech-Language-Hearing Association Leader 2003;8(10)6-21.

14. Altschuld JW, White JL. Needs Analysis: Analysis and Prioritization. 4th ed. Thousand Oaks, California: SAGE Publications Inc., 2010.

15. Cohen LJ. The Handy Psychology Answer Book. Canton: Visible Ink Press, 2011

16. Tye-Murray N. Foundations of Aural Rehabilitation: Children, Adults and Their Family Members. New York: Delmar, 2009.

17. Most T, Zaidman-Zait A. The needs of parents of children with cochlear implants. Volta Rev 2007;103:99-113.

18. Jackson CW, Turnbull AP. Impact of deafness on family life: A review of the literature. Topics Early Child Spec Educ 2004;24(1):15-29. DOI:10.1177/0271 1214040240010201

19. De Villiers D. Vocational training in school programs and occupational training at colleges in South Africa. American Annals of the Deaf 2010;155(4):504-506.

20. Hintermair M. Parental resources, parental stress, and socio-emotional development of deaf and hard of hearing children. J Deaf Stud Deaf Educ 2006;11(4):493-513. DOI:10.1093/deafed/enl005 